\title{
The Association of Crohn's Disease, Celiac Disease and Selective Ig A Deficiency
}

\author{
Ludmila Tankova $^{1}$, Vanya Gerova ${ }^{1}$, Plamen Getsov ${ }^{2}$, Nikolay Penkov ${ }^{3}$, Gergana Taneva ${ }^{1}$, \\ Ivan Terziev ${ }^{4}$, Radislav Nakov ${ }^{1, *}$ \\ ${ }^{1}$ Clinic of Gastroenterology, Clinical Center of Gastroenterology, University Hospital “Queen Joanna”, Sofia, Bulgaria \\ ${ }^{2}$ Department of Radiology, University Hospital “Queen Joanna”, Sofia, Bulgaria \\ ${ }^{3}$ Clinic of Abdominal Surgery, Clinical Center of Gastroenterology, University Hospital “Queen Joanna”, Sofia, Bulgaria \\ ${ }^{4}$ Department of Pathology, University Hospital “Queen Joanna”, Sofia, Bulgaria \\ *Corresponding author: radislav.nakov@gmail.com
}

\begin{abstract}
Concomitant occurrence of celiac disease (CeD) and Crohn's disease (CD) is rare and has been reported in several case reports and series. We report a case of a 63-year-old man with proximal CD, CeD and selective immunoglobulin (Ig) A deficiency (SIgAD). The patient was admitted in our Clinic for evaluation of epigastric pain, nausea, vomiting and weight loss (up to $7 \mathrm{~kg}$ ) appeared in the last 3 months. The imaging findings from abdominal ultrasound and CT enterography raised suspicion of a lymphoproliferative disease of the jejunum. To exclude the development of lymphoma based on long standing untreated atypical CeD, upper endoscopy with biopsies and serological tests were conducted, showing and subtotal villous atrophy and elevated levels of IgG tissue transglutaminase tTG. Histopathology documented CD (A3,L4,B2 according to Montreal's classification).
\end{abstract}

\section{Keywords: silent celiac disease, selective Ig A deficiency, Crohn's Disease}

Cite This Article: Ludmila Tankova, Vanya Gerova, Plamen Getsov, Nikolay Penkov, Gergana Taneva, Ivan Terziev, and Radislav Nakov, "The Association of Crohn's Disease, Celiac Disease and Selective Ig A Deficiency.” International Journal of Celiac Disease, vol. 4, no. 1 (2016): 30-33. doi: 10.12691/ijcd-4-1-8.

\section{Introduction}

Celiac Disease (CeD) is immuno-mediated systemic disease caused by gluten and similar prolamins in genetically susceptible individuals [1]. The disease may manifest with classic clinical presentation or with atypical symptoms, or even asymptomatic. CeD may by associated with other autoimmune diseases, immunodeficiencies and malignancies.

Selective immunoglobulin (Ig) A deficiency (SIgAD) is the most common primary immunodeficiency worldwide [2]. Patients with SIgAD have 10- to 20-fold increased risk of $\mathrm{CeD}$, and at least $2.6 \%$ of patients with $\mathrm{CeD}$ have SIgAD [3]. Patients suffering from SIgAD are often not aware of their IgA deficiency which may delay the diagnosis of $\mathrm{CeD}[4,5,6,7]$.

Crohn's disease (CD) is a chronic, relapsing inflammatory bowel disease that can affect any part of the gastrointestinal tract, most commonly ileum and colon. The CD pathogenesis has not been fully clarified, but currently it is assumed that the disease is a result of inappropriate immune response to intestinal microflora in genetically susceptible individuals [8].

Concomitant occurrence of $\mathrm{CeD}$ and $\mathrm{CD}$ is rare and has been reported in several case reports and series [9-15].

\section{Case Presentation}

A 63 years old man was admitted in our Clinic for evaluation of epigastric pain, nausea, vomiting and weight loss (up to $7 \mathrm{~kg}$ ) appeared in the last 3 months. His bowel habit was regular, without diarrhea or constipation. There was no history of fever, chills, night sweats, hematochezia, or melena. He had no past medical history of taking any medications within the last year. The patient has stopped smoking 3 months ago. He hasn't reported personal or familial history of any oncological or autoimminr diseases. Physical examination at admission revealed only a thin man with pallor of the skin; blood pressure - 130/70 mm Hg, without hepatosplenomegaly or peripheral lymphadenopathy. Initial laboratory tests showed iron deficiency anemia (hemoglobin: $109 \mathrm{~g} / \mathrm{L}$ [130-180 g/L] ; mean corpuscular volume: $75 \mathrm{fl}$ [82-98 fl]; iron: $4.6 \mu \mathrm{mol} / \mathrm{L}$ [6,6-26 $\mu \mathrm{mol} / \mathrm{L})$, $\mathrm{C}$ reactive protein: $9.44 \mathrm{mg} / \mathrm{dL}$ [0-0.8mg/L], sedimentation rate: $70 \mathrm{~mm}$ [up to $30 \mathrm{~mm} / \mathrm{h}$ ], albumin: $25 \mathrm{~g} / \mathrm{L}$ [35-50 g/L], fibrinogen: $569 \mathrm{mg} / \mathrm{dL}$ [200-340 mg/dL]. Immunlobulins serum concentrations showed SIgAD: Immunoglobulin A (Ig A): <0.05 g/L [0.9-3.5 g/L], Immunoglobulin G (Ig G): $13.48 \mathrm{~g} / \mathrm{L}$, Immunoglobulin M (Ig M): $2.6 \mathrm{~g} / \mathrm{L}$. Serum liver tests, bilirubin and renal function tests were in normal ranges. Stool examinations were negative for intestinal infections and parasitosis. Thyroid function tests and urine analysis were within the normal range. Human Immunodeficiency virus and Mantoux test were negative.

Abdominal ultrasonography showed thickened hypoechogenic small bowel wall up to $9 \mathrm{~mm}$ (Figure 1). Mild to moderate dilatation of small bowel loops with thickened Kerckring's folds were displayed (Figure 1). It was suspected lymphoproliferative small bowel disease or CD. Colonoscopy was normal up till the terminal ileum with normal ileocolonic histology. 


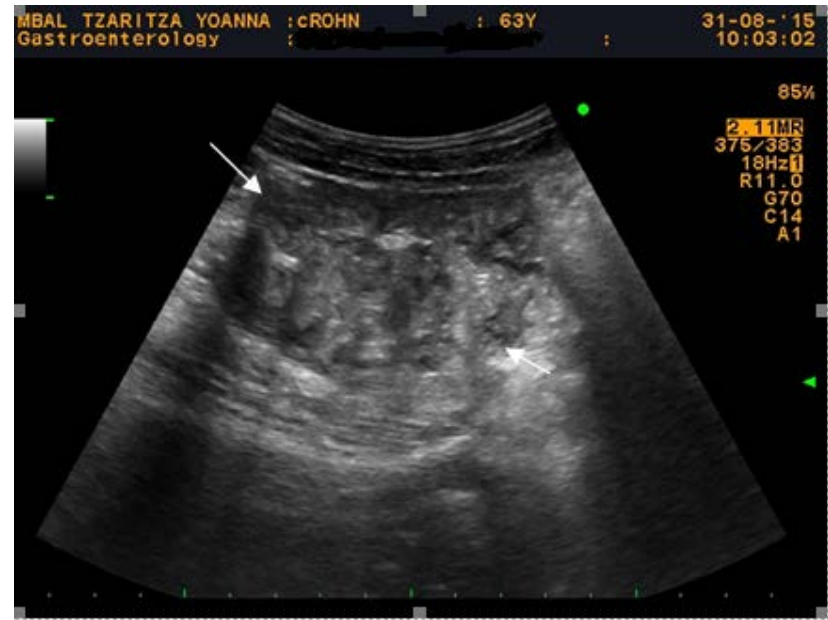

Figure 1. Abdominal ultrasound - thickened small bowel wall (up to 9 $\mathrm{mm}$ ) (white arrows). Small bowel lymphproliferative disorder was suspected

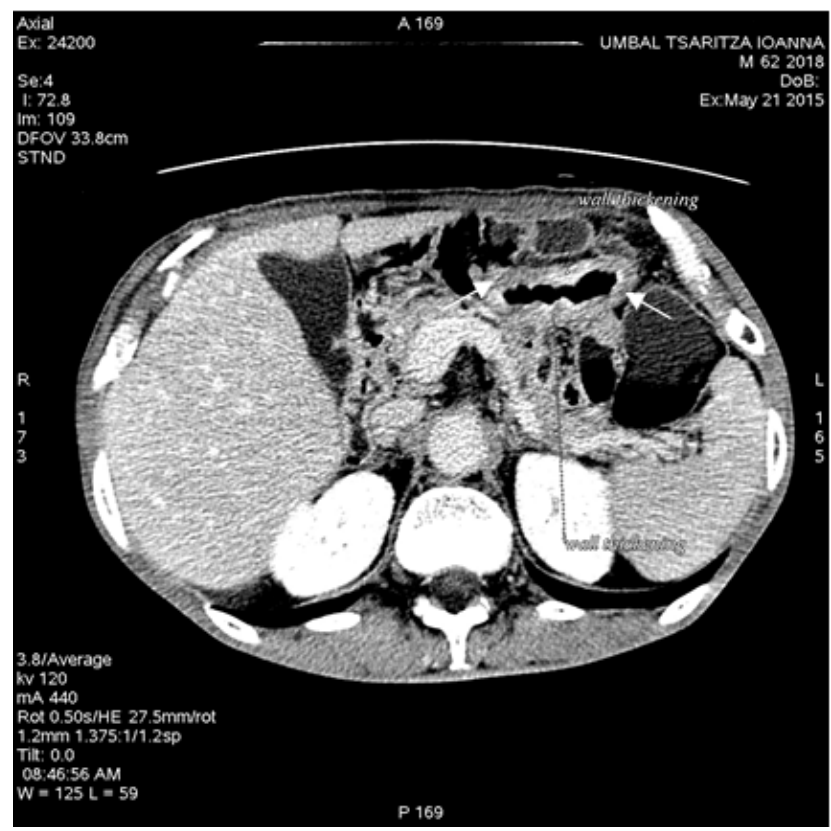

Figure 2. CT enterography - Concentric thickening of the jejunal wall up to $8-9 \mathrm{~mm}$ (white arrows)

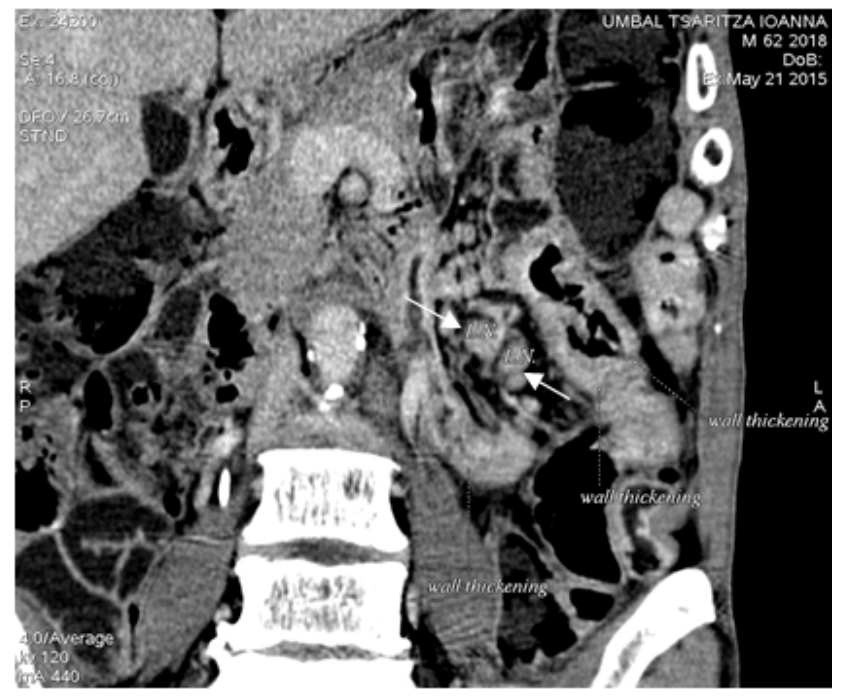

Figure 3. CT enterography - Two enlarged mesenteric lymph nodes (white arrows) are shown adjacent to the thickened small bowel wall

CT enterography showed a long segment of at least 30 $\mathrm{cm}$ of the jejunum with regular concentric thickened wall up to 8-9 mm (Figure 2). There was a slight prestenotic dilation. Multiple enlarged mesenteric lymph nodes were discovered (Figure 3). Data suggest firstly small bowel lymphoproliferative disorder.

To exclude the development of lymphoma, based on long standing untreated atypical CeD conventional serological tests and upper endoscopy with biopsies were conducted. Total Ig A serum concentration showed SIgAD. Serological tests for CeD were as follows: Anti tTG Ig A: $0.04 \mathrm{U} / \mathrm{ml}$ (normal < $15 \mathrm{U} / \mathrm{ml}$ ), Anti tTG Ig G: $84 \mathrm{U} / \mathrm{ml}$ (normal < $15 \mathrm{U} / \mathrm{ml}$ ), Anti-gliadin Ig G: $29 \mathrm{U} / \mathrm{ml}$. An upper endoscopy was performed and five duodenal biopsies were taken with two samples from the duodenal bulb.

Duodenal histology showed subtotal villous atrophy of the mucosa, crypt elongation with increased number of intraepithelial lymphocytes, which was consistent with untreated CeD (Marsh-Oberhuber's type IIIb changes) [16,17] (Figure 4).

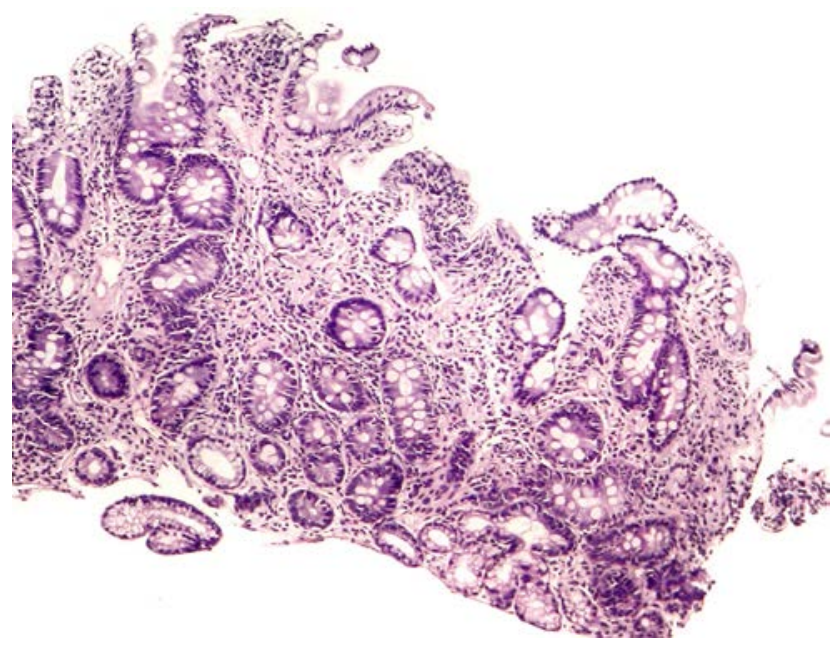

Figure 4. Histology of the duodenal mucosa. Subtotal villous atrophy with crypt hyperplasia and intraepithelial lymphocyte infiltration $(\times 10)$

Due to suspicion of enteropathy associated $\mathrm{T}$ cell lymphoma (EATL) in association with $\mathrm{CeD}$, as well as the need of histological verification, the patient was referred for exploratory laparotomy. During surgery a stricture in the proximal jejunum about $50 \mathrm{~cm}$ distal to the ligament of Treitz and multiple enlarged lymph nodes in the mesenteric area were found (Figure 5). Partial enteric resection with latero-lateral anastomosis was performed. Histological examination of the resected bowel segment showed ulcerated mucosa with inflammatory infiltrates in lamina propria, fibrosis of the submucosa and subserosa (Figure 6). Lymphoproliferative disease was excluded by a normal immunohistochemistry profile of the mucosal lymphocytes without any clonal proliferation. These findings according to the pathologist's opinion were consistent to CD.

The diagnosis of CD (A3, L4, B2 according to Montreal's classification) associated with $\mathrm{CeD}$ was established. The patient was prescribed a gluten-free diet and oral azathioprine at a dose of $2.5 \mathrm{mg} / \mathrm{Kg} / \mathrm{day}$ with iron and vitamin supplements. Nine months of follow-up he presented in a good condition, symptoms-free and reported a weight gain of $6 \mathrm{~kg}$. Hematological and biochemical tests were in normal range and anti tTG Ig G disappeared. HLA tests for CeD or other genetic analysis (incl. NOD2 mutation for CD) were not performeddue to 
financial reasons as our healrhcare insurance fund does not cover the costs of genetic tests.

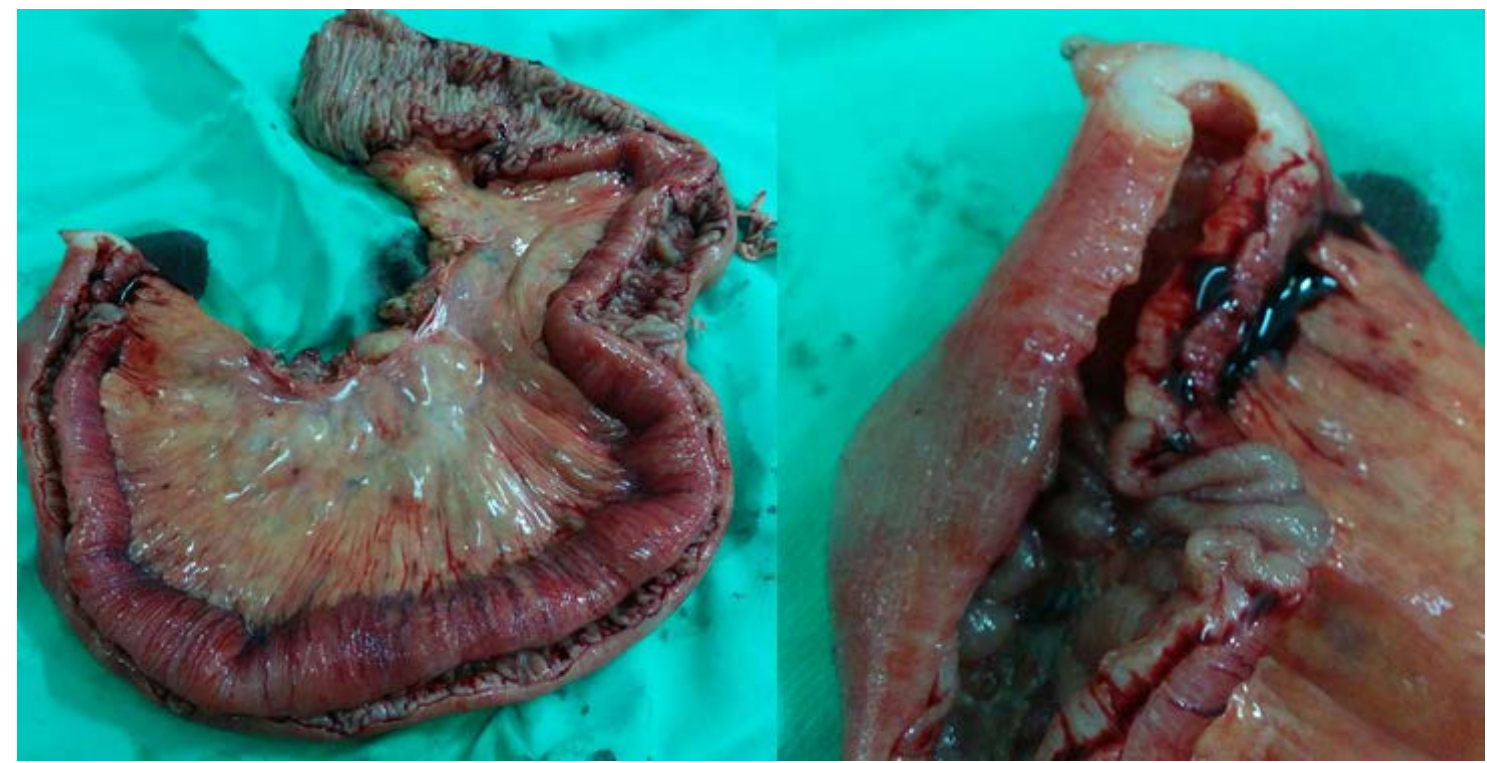

Figure 5. Macroscopic finding of the resected jejunal segment $-50 \mathrm{~cm}$ long segment with thickened wall and strictute in the proximal region. Wall thickness is up to $1 \mathrm{~cm}$

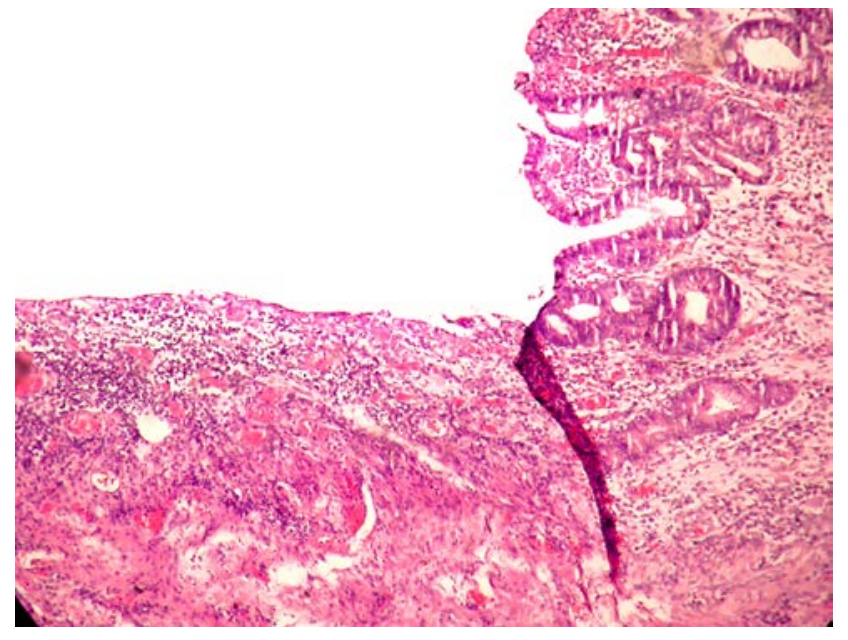

Figure 6. Histology of the resected jejunal segment - ulcerated mucosa with inflammatory infiltrates in lamina propria, fibrosis of the submucosa and subserosa

\section{Discussion}

CeD and CD are autoimmune conditions that affect the digestive system and share many symptoms.

$\mathrm{CeD}$ is more common in women. CD affects both genders similarly, most often between the second and fourth decade of life. The combination of both diseases is more common in young males. The upper GI tract location of CD is also more often in young patients. Only 10-15\% of the cases with CD appear in patients older than 60 years [18]. In the majority of the published cases, CeD preceded the diagnosis of CD [11,12,19]. Furthermore, most patients had symptomatic $\mathrm{CeD}$ and colonic or terminal ileum CD [20,21].

Our clinical case was interesting at least for several reasons - association of $\mathrm{CeD}$ with $\mathrm{CD}$; the proximal location of $C D$; the older age of the patient and the presence of SeIgAD

Perhaps the combination of $\mathrm{CeD}$ and $\mathrm{CD}$ is actually more common. Indeed, Tursi at al. in one prospective study establish high prevelance of $\mathrm{CeD}$ in a consecutive series of patients affected by CD patients (almost 26\%) [15]. Probably due to the atypical clinical course in the most of patients sometimes CeD remains undiagnosed. In our case, the detection of the disease is due to the suspicion of small intestine lymphoma, which may be associated with untreated CeD or with CD.

Our patient presented with similar symptoms for both diseases probably after a long subclinical course. Both diseases share an olidosymptomatic mode of presentation with nonspecific overlapping symptoms. The late manifestation of CD may indicate the silent character of $\mathrm{CeD}$ as no clinical presentation has been obsereved for many years. Although both diseases were diagnosed simultaneously, it was unclear which of them had developed earlier. CT enterography findings for jejunal stenosis suggested the longer CD duration. It is not clear why this patient has not had any clinical symptoms for a long time.

The association between $\mathrm{CeD}$ and SIgAD complicates serological testing for CeD [7]. Indeed, routine serology of $\mathrm{CeD}$ is IgA-based and is expected to be negative in IgAdeficiency as in our patient. Diagnosis of $\mathrm{CeD}$ was confirmed by the positive IgG anti tTG antibodies and histological findings of subtotal villous atrophy from multiple duodenal biopsies.

The association of CD and CeD may be explained by shared genetic predisposition, increased gut permeability to antigens observed in both diseases [7,9,10,14,15,20].

The present and similar clinical cases have described in the medical literature raise in testing for $\mathrm{CeD}$ in patients with $\mathrm{CD}$, although currently there is not enough evidence to recommend routine serological screening for $\mathrm{CeD}$ in CD patients $[7,15]$.

\section{Conclusion}

Our case report is of interest because of a very rare combination of silent $\mathrm{CeD}$, SIgAD and active $\mathrm{CD}$ of the 
upper gastrointestinal tract with stricturing behavior. Moreover, all these disorders were diagnosed at the same time in 63-year-old man.

\section{References}

[1] Martucci, S., Biagi, F., Di Sabatino, A. and Corazza, G.R., “Coeliac disease," Dig Liver Dis, 34 (2). S150-S153. Sep 2002.

[2] Yel, L., "Selective IgA deficiency," J Clin Immunol, 30 (1). 10-16. Jan 2010.

[3] Cataldo, F., Marino, V., Ventura, A., Bottaro, G., Corazza, G.R. and the Italian Society of Paediatric Gastroenterology and Hepatology (SIGEP), and "Club del Tenue” Working Groups on Coeliac Disease, "Prevalence and clinical features of selective immunoglobulin A deficiency in coeliac disease: an Italian multicentre study,” Gut. 42 (3). 362-365. Mar 61998.

[4] Rostom, A., Dube, C., Cranney, A., Saloojee, N., Sy, R., et al, "The diagnostic accuracy of serologic tests for $\mathrm{CeD}$ : a systematic review," Gastroenterol, 128 (4). S38-46. Apr 2005.

[5] van der Windt, D.A., Jellema, P., Mulder, C.J., Kneepkens, C.M. van der Horst, H.E., "Diagnostic testing for CeD among patients with abdominal symptoms: a systematic review," JAMA, 303 (17). 1738-1746. May 2010.

[6] Agarwal, S. and Mayer, L, "Diagnosis and Treatment of Gastrointestinal Disorders in Patients With Primary Immunodeficiency,“ Clin Gastroenterol Hepatol., 11(9). 10501063. Sep 2013.

[7] Rubio-Tapia, A., Hill I.D., Kelly, C.P., Calderwood, A.H. and Murray, J.H., "American College of Gastroenterology Clinical Guideline: Diagnosis and Management of CeD," $A m \quad J$ Gastroenterol, 108 (5). 656-676. May 2013.

[8] Podolsky, D.K, "Inflammatory bowel disease, “ N Engl J Med, 347 (6). 417-429. Aug 2002.

[9] Masachs, M., Casellas, F. and Malagelada, R, "Inflammatory bowel disease in celiac patients," Rev Esp Enferm Dig, 99 (8). 446-450. Aug 2007.
[10] Schedel, J., Rockmann, F., Bongartz, T., et al, “Association of Crohn's disease and latent CeD: a case report and review of the literature,” Int J Colorectal Dis, 20 (4). 376-380. Dec 2004.

[11] Kitis, G., Holmes, G.K., Cooper, B.T., Thompson, H. and Allan. R.N., "Association of CeD and inflammatory bowel disease," Gut, 21 (7). 636-641. Jul 1980.

[12] Curtis, W.D., Schuman, B.M. and Griffin, J.W., "Association of gluten-sensitive enteropathy and Crohn's colitis," Am J Gastroenterol, 87 (11). 1634-1637. Nov 1992.

[13] Karoui, S., Boubaker, J., Hamzaoui, S., et al., "Association of asymptomatic CeD and Crohn's disease (article in french)," Ann Med Interne (Paris), 151 (5). 411-412. Sep 2000.

[14] Lezzi, L., Medeiros, B., Feitosa, M. et al., "Association between $\mathrm{CeD}$ and Crohn's disease - a challenge to the coloproctologist," J Coloproctol, 32 (3). 329-333. 2012.

[15] Tursi, A., Giorgetti, G., Brandimarte, G. and Elisei W, "High prevelance of CeD among patients affected by Crohn's disease," Inflamm Bowel Dis, 11 (7). 662-666. Jul 2005.

[16] Marsh, M.N., "Gluten, major histocompatibility complex and the small intestine. A molecular and immunobiologic approach to the spectrum of gluten sensitivity (celiac sprue),“ Gastroenterology, 102 (1). 330-54. Jan 1992.

[17] Oberhuber, G., Granditsch, G. and Vogelsang, H., "The histopathology of coeliac disease: time for a standardized report scheme for pathologists,” Eur J Gastroenterol Hepatol, 11 (10). 1185-94. Oct 1999.

[18] Gisbert, J.P. and Chaparro M., "Systematic review with metaanalysis: inflammatory bowel disease in the elderly," Aliment Pharmacol Ther, 39 (5). 459-477. Mar 2014.

[19] Patel, J., Agasti, A., Rao, S., et al., "CeD preceding Crohn's disease,” Trop Gastroenterol., 32(3). 236-238. Jul 2011.

[20] Yang, A., Chen, Y., Scherl, E. et al., "Inflammatory bowel disease in patients with CeD,” Inflamm Bowel Dis, 11 (6). 528-532. Jun 2005.

[21] Charif, M., Abkari, A., Ibrahimi, M. Y., "The association of Crohn's disease with CeD,” OJGAS, 2 (4). 184-186. Nov 2012. 